Article

\title{
Novel Semi-Parametric Algorithm for Interference-Immune Tunable Absorption Spectroscopy Gas Sensing
}

\author{
Umberto Michelucci ${ }^{1, \dagger}$ and Francesca Venturini ${ }^{2, *,+}$ \\ 1 Udata.Science, Dübendorf 8600, Switzerland; um@udata.science \\ 2 Institute of Applied Mathematics and Physics, Zurich University of Applied Sciences, \\ Winterthur 8401, Switzerland \\ * Correspondence: vent@zhaw.ch \\ + These authors contributed equally to this work.
}

Received: 10 September 2017; Accepted: 4 October 2017; Published: 7 October 2017

\begin{abstract}
One of the most common limits to gas sensor performance is the presence of unwanted interference fringes arising, for example, from multiple reflections between surfaces in the optical path. Additionally, since the amplitude and the frequency of these interferences depend on the distance and alignment of the optical elements, they are affected by temperature changes and mechanical disturbances, giving rise to a drift of the signal. In this work, we present a novel semi-parametric algorithm that allows the extraction of a signal, like the spectroscopic absorption line of a gas molecule, from a background containing arbitrary disturbances, without having to make any assumption on the functional form of these disturbances. The algorithm is applied first to simulated data and then to oxygen absorption measurements in the presence of strong fringes.To the best of the authors' knowledge, the algorithm enables an unprecedented accuracy particularly if the fringes have a free spectral range and amplitude comparable to those of the signal to be detected. The described method presents the advantage of being based purely on post processing, and to be of extremely straightforward implementation if the functional form of the Fourier transform of the signal is known. Therefore, it has the potential to enable interference-immune absorption spectroscopy. Finally, its relevance goes beyond absorption spectroscopy for gas sensing, since it can be applied to any kind of spectroscopic data.
\end{abstract}

Keywords: interference; interference cancellation; noise reduction; digital filtering; spectroscopy; sensors

\section{Introduction}

Due to the enormous progress in availability and performance of laser light sources and electro-optical components, tunable diode laser absorption spectroscopy (TDLAS) has entered various disciplines both in research and industrial applications. Being a highly-sensitive, selective, fast, non-destructive and in situ method, TDLAS is currently more and more used for quantitative assessment of gas concentration in several fields. These include, to mention only a few, atmospheric environmental monitoring [1-6], medical diagnostics [7-9], chemical analysis [10], and industrial process control [11-13]. The increasing number of applications has pushed the requirements for this method both in terms of sensitivity and in terms of stability. On the other hand, for practical and commercial applications, there is a growing interest in compact, simple in design and cost-effective sensitive sensors that do not require special optical components but guarantee the sensitivity achievable with complex laboratory equipment.

One of the most common limits to the sensor performance is the presence of unwanted interference fringes due to etalons [14]. These interferences may arise due to multiple reflections from reflecting or 
scattering surfaces in the system, like mirrors, lenses, optical fiber end faces, laser-head windows or dust particles in the gas [14]. Even surfaces that are diffusive, for example due to dust deposited on sensor windows, can give rise to fringes over time [15]. In particular, fringes that have a free spectral range (FSR) on the order of the width of the absorption lines contribute to significant errors in the determination of the line features. Special strategies have been proposed to deal with these types of interferences, as, for example, in [16], where for small fringe amplitudes and a measurement window that is a multiple of the fringe FSR, the fringe was removed by an analysis in the Fourier domain. Furthermore, since the amplitude and the frequency of these interferences depend on the distance and alignment of the optical elements, they are affected by temperature changes and by mechanical disturbances, giving rise, for example, to a drift of the output signal, thus worsening the long-term performance of the system [17].

The simplest strategy to reduce the effects of interference fringes consists of using anti-reflection coating and wedging or angling of the optical surfaces. Other approaches include, to mention only few, dithering one of the surfaces creating the interference and integrating the signal so as to average out its influence [18,19], selecting a particular modulation frequency [20] or modulation scheme [21,22], specifically choosing the distance between the interfering surfaces [23], and post-processing filtering [24]. A comprehensive review on signal enhancement and noise reduction techniques can be found in [25]. Although all of these approaches have been successfully implemented in past years, they nonetheless have limitations in the practical implementation depending on the application-specific conditions. For example, it may not always be possible to dither one surface, and the application of a specific detection scheme may limit the flexibility of the measurement.

In this work, a new and widely usable approach is presented that relies only on post processing of the data. Therefore, it requires no modification to the apparatus setup or hardware, and can be easily adapted to different experimental configurations. The new presented algorithm allows the extraction of a signal, as the absorption lines of a gas molecule, from a background containing arbitrary disturbances without having to make any assumption on the characteristics of these disturbances in terms of functional form. Therefore, it has the potential to improve the sensitivity and the stability of TDLAS. Furthermore, this algorithm, which is particularly easy to implement if the Fourier transform of the signal can be written in closed form, is very general and can be applied to any kind of spectroscopic data. The paper is organized as follows: Section 2 describes the fundamentals of the method and of the algorithm; Section 3 demonstrates its application to simulated signals; and Section 4 shows the results for a case of direct absorption spectroscopy for oxygen gas sensing.

\section{Description of the Algorithm}

The algorithm described in this work has the ability to identify a spectroscopic feature from an arbitrary background that does not need to be modeled. The total signal detected in an experiment, here referred to as "total signal" $I_{t o t}(x)$, is modeled as a sum of two contributions: one spectroscopic feature, like an absorption line, referred to here as "signal" $I(x)$ and a background referred to here as "background" $B(x)$ :

$$
I_{t o t}(x)=I(x)+B(x) .
$$

In the case of direct absorption spectroscopy, $I(x)$ is the absorbance and $I_{t o t}(x)$ is the distorted absorbance due to $B(x)$. As mentioned above, the method shines if the background $B(x)$ cannot be modeled by a known analytical expression. In fact, if $B(x)$ is not known, it is not possible to perform a nonlinear fit of $I_{t o t}(x)$ and extract the signal $I(x)$ without making assumptions on the functional form of $B(x)$. On the other hand, if the functional form is known but very complex, the algorithm may be advantageous because the inclusion of the background in the nonlinear fit may not be possible. Another significant advantage of the proposed algorithm is that the extraction works equally well independently of the amplitude of the interferences, as it will be shown in Sections 3 and 4. 


\subsection{Steps of the Algorithm: General Description}

Before describing the steps of the algorithm, the nomenclature and hypothesis for its applicability are introduced. The algorithm is based on the main hypothesis that the Fourier transform of the background $B(x)$ is significantly different than zero only for values of $k$ smaller than a certain cut-off $k_{0}$

$$
F\left(I_{\text {tot }}\right)(k)=\left\{\begin{array}{l}
F(I)(k)+F(B)(k) \text { for }|k|<k_{0} \\
F(I)(k)+\epsilon \text { for }|k|>k_{0}
\end{array}\right.
$$

where $F(\cdot)$ denotes the continuous Fourier transform (CFT), $k_{0}$ a cut-off frequency and $\epsilon$ contains the contribution of $F(B)(k)$ for $|k|>k_{0}$, which is assumed to be negligible. One central aspect is the determination of a reasonable estimate for this cut-off frequency, as described in the section "Determine the cut-off frequency".

Note that this formulation applies to continuous functions. Since in practice there will always be only a discrete set of points, it is necessary to approximate the CFT in Equation (2) by a modified discrete Fourier transform (DFT)

$$
D_{i}\left(I_{t o t}\right)=\left\{\begin{array}{l}
D_{i}(I)+D_{i}(B) \text { for }|i|<i_{0}, \\
D_{i}(I)+\epsilon \text { for }|i|>i_{0},
\end{array}\right.
$$

where $D_{i}(\cdot)$ denotes the modified DFT defined in Appendix A Equation (A6), $i_{0}$ the cut-off point corresponding to the cut-off frequency $k_{0}$, and $\epsilon$ contains the contribution of $D_{i}(B)$ for $|i|>i_{0}$, which is assumed to be negligible.

The schematic flow diagram of the algorithm is shown in Figure 1 to give the reader a high-level understanding of the idea behind it. The single steps are described in detail below.

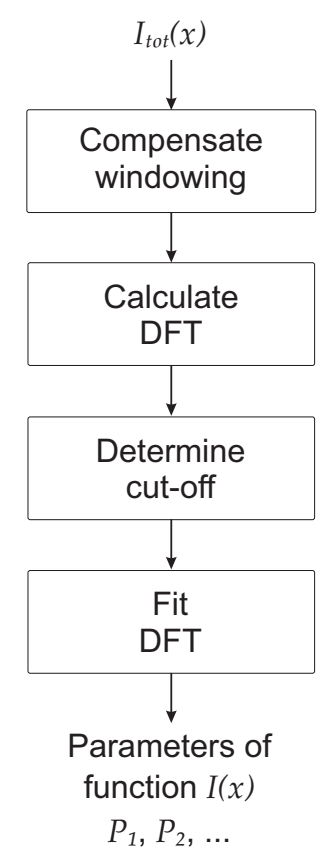

Figure 1. Schematic flow diagram of the steps of the algorithm to extract a signal $I(x)$ from a total signal $I_{t o t}(x)$.

\subsection{Compensate Windowing}

In all experiments, the data always cover a limited range in the $x$-direction. For example, the data shown in this paper are measured for a finite laser wavelength range. Mathematically, this is 
equivalent to applying a rectangular window (RW) before calculating the Fourier transform. This is a problem that, if not addressed, will limit the precision that can be achieved with the described algorithm. In fact, the RW results in making $\epsilon$ in Equation (2) not negligible anymore, and thus leads to an error in the fitting procedure of $|F(I(x))(k)|$. This is due to the fact that the DFT of a RW has an amplitude envelope that is proportional to $1 / k$, and so it does not go to zero fast enough [26].

To reduce the effect of windowing considerably, the proposed algorithm applies a more intelligent window. The not so often used Tukey window $[27,28]$ has remarkable properties that help tremendously in reducing $\epsilon$ dramatically. The Tukey window, indicated with $T(x)$, is a perfectly flat (constant) symmetric function in the middle that then decreases rapidly to zero on the sides.

The width of the constant part of the Tukey function has to be chosen intelligently. Figure 2 shows a Lorentzian function with a half width at half maximum (HWHM) indicated as $P_{2}$ and Tukey function with a width indicated as $W$, with both functions normalized to 1 for clarity. In this work, a.u. indicates arbitrary units. As it is easy to understand from Figure 2, if $W$ is significantly bigger than $P_{2}$, then $T(x) I(x)$ can be approximated with $I(x)$. Therefore, defining $\tilde{I}_{t o t}(x)=T(x) I_{t o t}(x)$, it follows that

$$
\tilde{I}_{t o t}(x)=T(x) I(x)+T(x) B(x) .
$$

This is the function of which the DFT has to be calculated, instead of simply using $I_{t o t}(x)$. Equation (4) can then be approximated under the assumption of $W$ being significantly bigger than $P_{2}$ as

$$
\tilde{I}_{t o t}(x)=I(x)+T(x) B(x) .
$$

$\tilde{I}_{t o t}(x)$ is thus the sum of the signal of $I(x)$ and a background that is the product of the original background $B(x)$ and $T(x)$. The modified background $T(x) B(x)$ has a Fourier transform that goes to zero much more rapidly $([27,28])$. This means that $\epsilon$ is much smaller if one considers $\tilde{I}_{t o t}(x)$ instead of $I_{t o t}(x)$. In other words, while using $\tilde{I}_{t o t}(x), \epsilon$ will contain the contribution of $D_{i}(T \cdot B)$ that is considerably smaller than $D_{i}(B)$ multiplied by an RW.

Analyzing the deviation between the output of the algorithm and the input signal $I(x)$ with simulated data, it was established that the algorithm works well if the width of the Tukey window is $W \gtrsim 20 P_{2}$. In this work, $W=20 P_{2}$ was used for both the simulated and for the experimental data. The case with $W=20 P_{2}$ is shown schematically in Figure 2 .

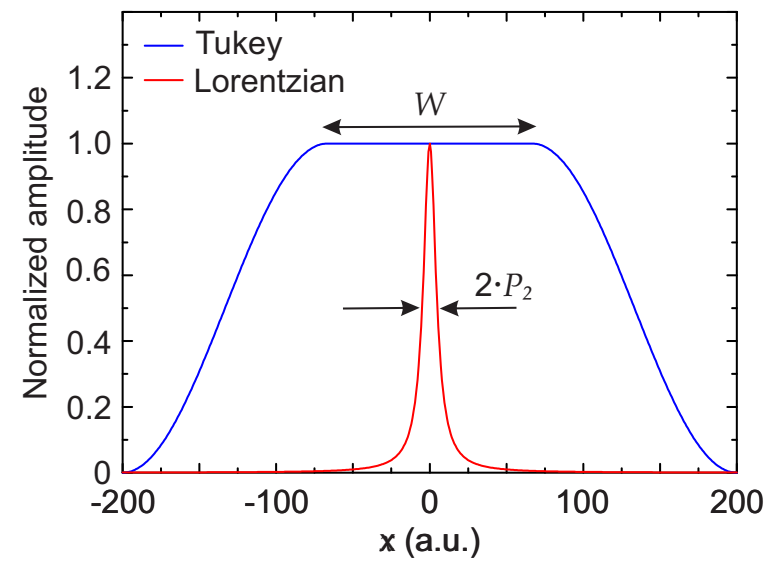

Figure 2. Schematic representation on how to choose the width of the Tukey window $W$ compared to the Lorentzian HWHM $P_{2}$. Here, it is $W=20 P_{2}$.

\subsection{Calculate the DFT}

The step after compensating for the windowing is the calculation of the DFT. To be able to extract the parameters of the function $I(x)$ directly from the DFT, it is essential to approximate the CFT by a 
modified DFT as described in Appendix A. For all the data shown in this paper, the DFT was calculated using the formula (A6).

\subsection{Determine Cut-Off}

As shown in Figure 1, the next step is to determine the optimal cut-off point $i_{0}$ that plays an important role and needs to be chosen carefully. The approach proposed in this work is to choose $i_{0}$ so as to maximize the coefficient of determination $R^{2}$ obtained by fitting the DFT for $i>i_{0}$ to the functional form of the Fourier transform of the line shape. In our example for a Lorenztian function, the DFT was fitted by Equation (7), as is explained in more detail later. An example of the implementation is described in the following algorithm written in pseudo code, where $\mathrm{N}$ indicates the number of points of the dataset (i.e., the finite number of experimental points), $D_{-} i$ the ith point of the DFT of the signal $\tilde{I}_{t o t}(x)$, Rsquared the coefficient of determination $R^{2}$, FFFT the functional form of the Fourier transform of $I(x)$, and Rlimit the value which should be reached for $R^{2}$. This limiting value can be helpful since, beyond a certain value, even if $R^{2}$ continues to increase when increasing $i_{0}$, the quality of the fit will not improve significantly and it is not necessary to exclude more points from the DFT for the fit:

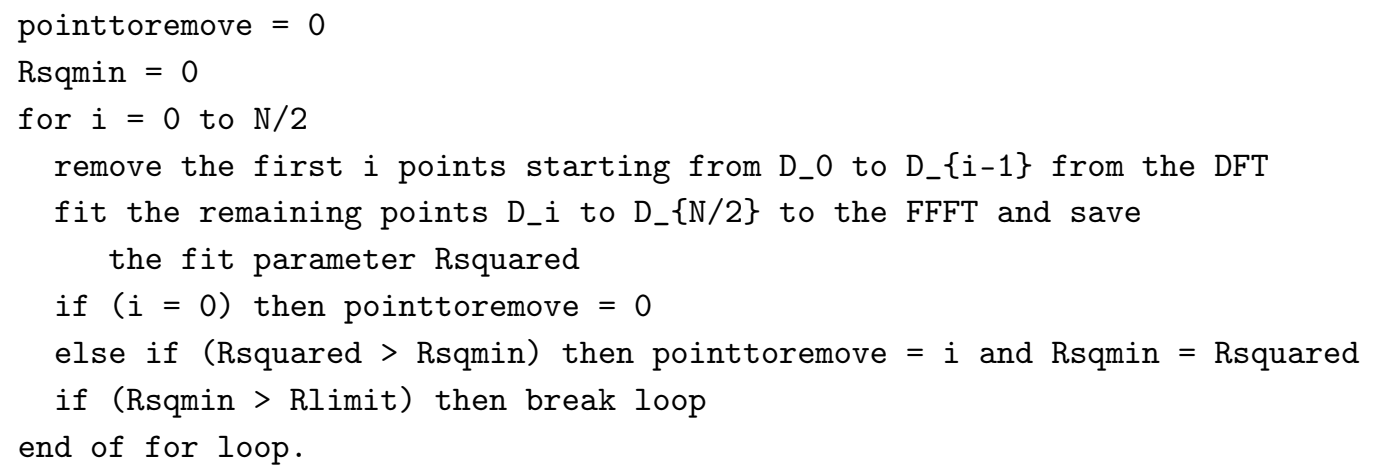

At the end of this loop in the abovementioned pseudo-code $i_{0}$ is determined and saved in the variable pointtoremove. Rsquared can be chosen depending on the application. For the curves shown in this paper, the loop was stopped for Rlimit $=0.99999$. An example of the evolution of $R^{2}$ with increasing $i_{0}$ is shown in Figure 3. The data refer to the third scenario described later in Section 3. Above $i_{0}=30, R^{2}$ still continues to slightly increase, but the statistical goodness of the fit does not improve further.

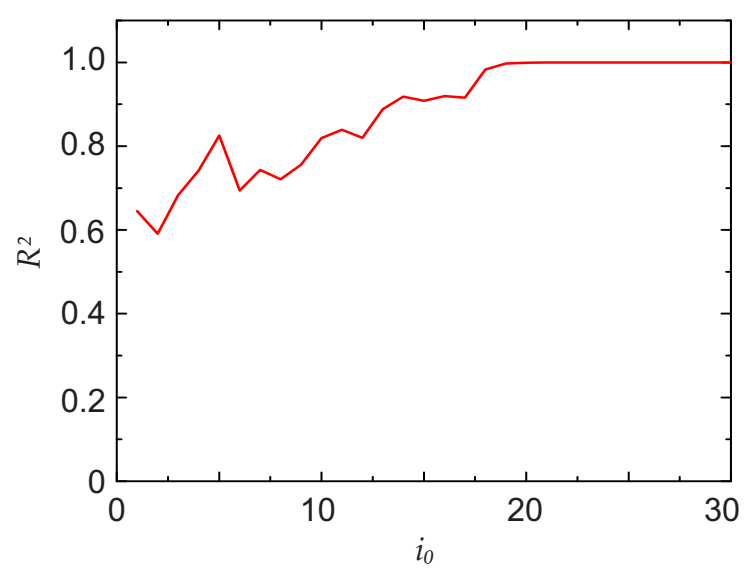

Figure 3. Evolution of $R^{2}$ with increasing value of the cut-off $i_{0}$. The data correspond to the third scenario described in Section 3. 


\subsection{Fit of DFT}

The final step of the algorithm is to perform a nonlinear fit of the DFT for $i>i_{0}$. Since the functional form of the DFT is known, this is a standard procedure that can be performed, for example, with least-square-fit routines and will not be discussed here. After the fit $I(x)$ is determined without the need of doing an inverse Fourier transform since the functional form of $|F(I(x))(k)|$ is known.

\subsection{Algorithm Applied to a Lorentzian Line Shape}

In this section, the implementation of the algorithm for a Lorentzian signal $I(x)$ is described as an example. This functional form was chosen because it describes the absorption lines of many gas molecules, as, for example, oxygen under atmospheric conditions. In other conditions, like at higher temperatures or lower pressures, the Gaussian contribution due to Doppler broadening cannot be neglected and a Voigt profile is a better description.

The Lorentzian function can be written as

$$
I(x)=\frac{P_{1} P_{2}}{\pi\left(x^{2}+P_{2}^{2}\right)}
$$

with $P_{1}, P_{2}>0$. In this form, $P_{1}$ and $P_{2}$ represent the area and the HWHM of the line. Writing $I(x)$ in this form is particularly advantageous since in direct absorption spectroscopy the gas concentration can be determined directly from the area under the line, and is thus directly proportional to $P_{1}$. In this formulation, $|F(I(x))(k)|$ is then a simple exponential

$$
F(I(x))(k)=P_{1} e^{-P_{2}|k|} .
$$

Thus, once the parameters $P_{1}$ and $P_{2}$ are determined from the fit of the DFT, $I(x)$ is known.

\section{Application to Simulated Data}

The novel algorithm was first applied to artificially simulated data to demonstrate its functioning and its performance. Since the signal to be extracted $I(x)$ is known, it is possible to estimate the accuracy and robustness of the algorithm in the presence of backgrounds with different characteristics. In particular, three scenarios with different types of periodic disturbances were simulated. The signal to be extracted, $I(x)$, is for all three cases the same Lorentzian function written in the form of Equation (6) with $P_{1}=5 \pi$ and $P_{2}=5$. All three scenarios were chosen to reflect real cases that are typical of TDLAS.

The first scenario is chosen to represent the experimental situation when the background has a periodic disturbance with an FSR comparable to the width of the line to be detected. This type of background is particularly problematic because it strongly affects the determination of the line shape. Furthermore, it cannot be removed by introducing a small jitter on the diode laser current and averaged out [29] or be filtered out with standard post-processing methods, without introducing a significant distortion of the line shape. This type of background is taken here as a simple a cosine function

$$
B(x)=0.07 \cos (0.1 x+1) .
$$

The total signal $I_{t o t}(x)$ in this case, together with the expected signal $I(x)$ are shown in Figure 4a. Also shown is the result obtained by applying the described algorithm. Despite the problematic background, the output of the algorithm is practically identical to $I(x)$. The percent deviation of the two parameters $P_{1}$ and $P_{2}$ describing the Lorentzian obtained with the algorithm from the initial value used to generate $I(x)$ is only of $0.27 \%$ for $P_{1}$ and $0.23 \%$ for $P_{2}$. 

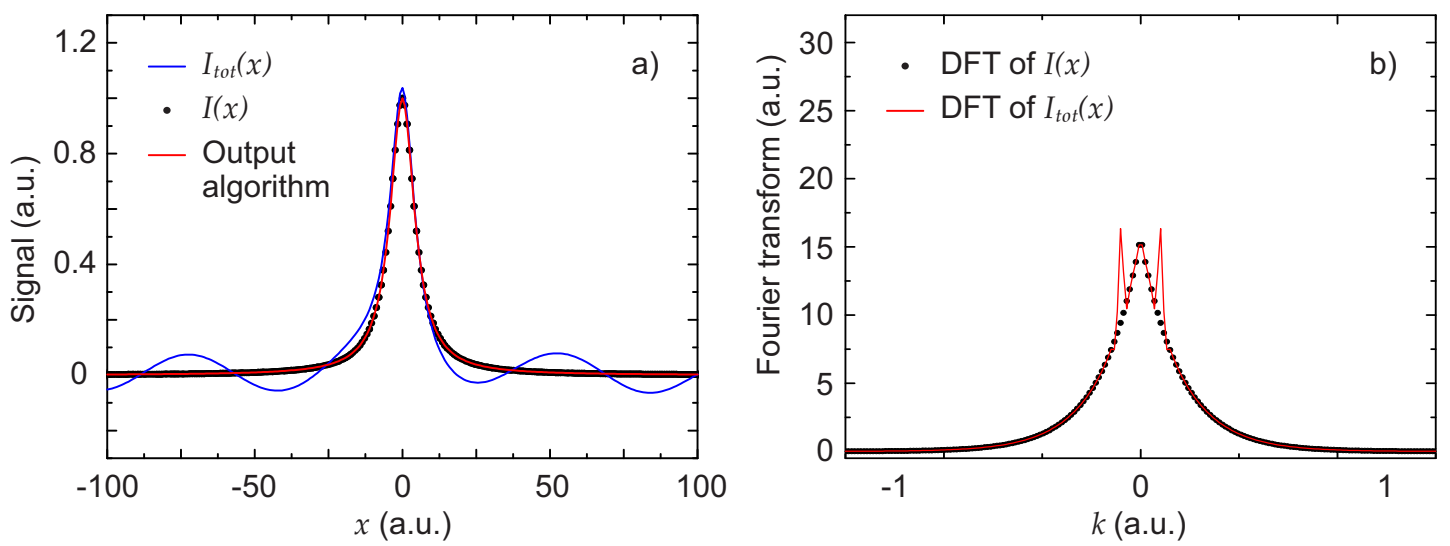

Figure 4. First scenario: disturbance with an FSR comparable to the line width. (a) simulated experimental total signal $I_{t o t}(x)$ (blue line), Lorentzian line shape signal $I(x)$ (black dots) and extracted signal obtained with the algorithm (red line); (b) DFT of the total signal $\left|F\left(I_{\text {tot }}(x)\right)(k)\right|$ (red line) and DFT of the Lorentzian signal $|F(I(x))(k)|$ (black points).

To better illustrate the contribution of the background, the DFT of the Lorentzian and the DFT of the total signal signal are also shown in Figure $4 \mathrm{~b}$. The two peaks visible in the figure represent the contribution of the background. With the pseudo-code algorithm described in Section 2.1, the cut-off was $i_{0}=13$.

The second scenario discussed here is that of a background with a weak disturbance characterized by an FSR much larger than the line width and as large as or larger than the measuring range. This type of disturbance arises because of reflections between two surfaces separated by a very short physical dimension, like the laser-chip output face and the glass window of the laser packaging. The chosen functional form to simulate this scenario is the following:

$$
B(x)=0.07 \cos (0.02 x+1.6) .
$$

The result of the algorithm for this scenario is shown in Figure 5. Figure 5a plots the total signal $I_{t o t}(x)$, the expected signal $I(x)$ and the result obtained by applying the algorithm. Figure $5 \mathrm{~b}$ shows the DFT of the Lorentzian and the DFT of the total signal. The two peaks, due to the contribution of the background, are now very close to zero, which makes the extraction particularly unproblematic. With the pseudo-code algorithm described in Section 2.1, the cut-off was $i_{0}=7$.
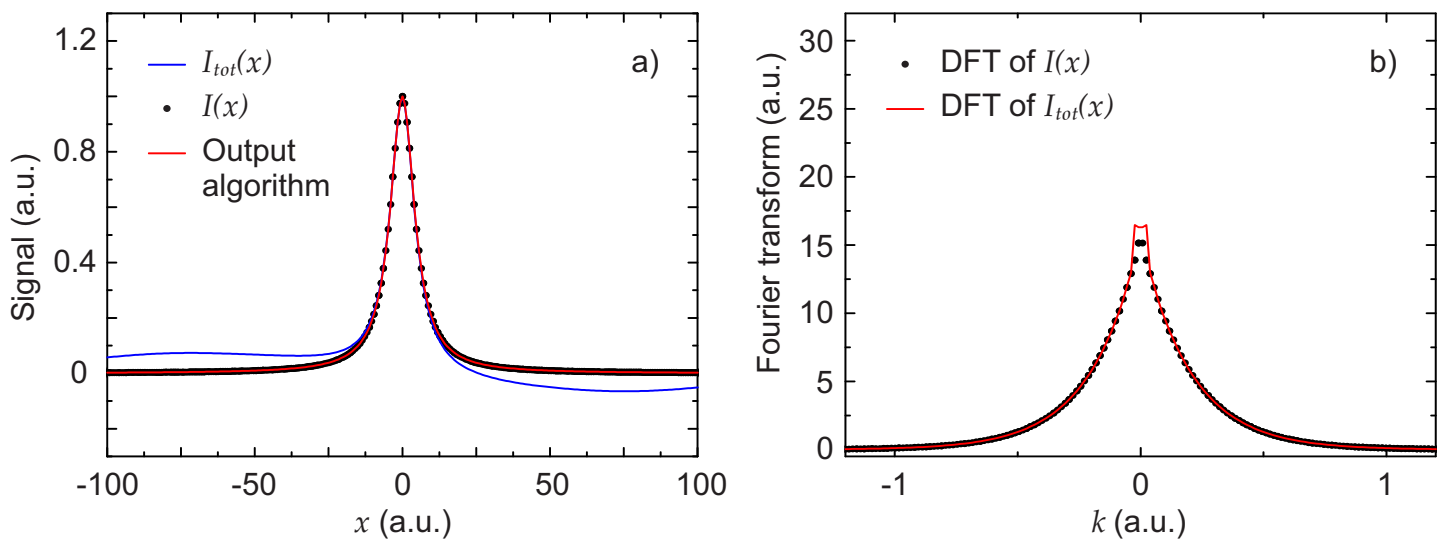

Figure 5. Second scenario: weak disturbance with an FSR as large as the measuring window. (a) simulated experimental total signal $I_{\text {tot }}(x)$ (blue line), Lorentzian line shape signal $I(x)$ (black dots) and extracted signal obtained with the algorithm (red line); (b) DFT of the total signal $\left|F\left(I_{\text {tot }}(x)\right)(k)\right|$ (red line) and DFT of the Lorentzian signal $|F(I(x))(k)|$ (black points). 
In this case, it is also clear from the figure that the algorithm extracts the signal exceedingly well. The percent deviation of the two parameters describing the Lorentzian obtained with the algorithm from the expected values is only of $0.28 \%$ for $P_{1}$ and $0.25 \%$ for $P_{2}$.

As a third scenario, a background resulting from the sum of a hundred cosine functions is considered. This less realistic case is chosen to demonstrate that, no matter how dramatic the interferences are, the algorithm can extract signal $I(x)$ very well. In addition, this scenario illustrates the case when the functional form of the background is too complex to be included in a nonlinear fit of $I_{t o t}(x)$. The background is thus written as

$$
B(x)=\sum_{i=1}^{100} A_{i} \cos \left(w_{i} x+\phi_{i}\right)
$$

where $w_{i}$ is chosen randomly from a normal distribution with an average equal to zero and a standard deviation of $0.1, \phi_{i}$ from a normal distribution with an average equal to zero and a standard deviation of 0.2 , and $A_{i}$ from a normal distribution with an average equal to zero and a standard deviation of 0.03 .

Figure 6a shows the total signal $I_{t o t}(x)$, the expected signal $I(x)$ and the result obtained by applying the described algorithm. Despite the very complicated background, the extraction of the signal $I(x)$ by the algorithm works very well. In Figure $6 \mathrm{~b}$, the DFT of the Lorentzian and the DFT of the total signal are also shown. Due to the high number of cosine functions in the background, the DFT has a very structured shape. With the pseudo-code algorithm described in Section 2.1, the cut-off was $i_{0}=30$ (see also Figure 3).
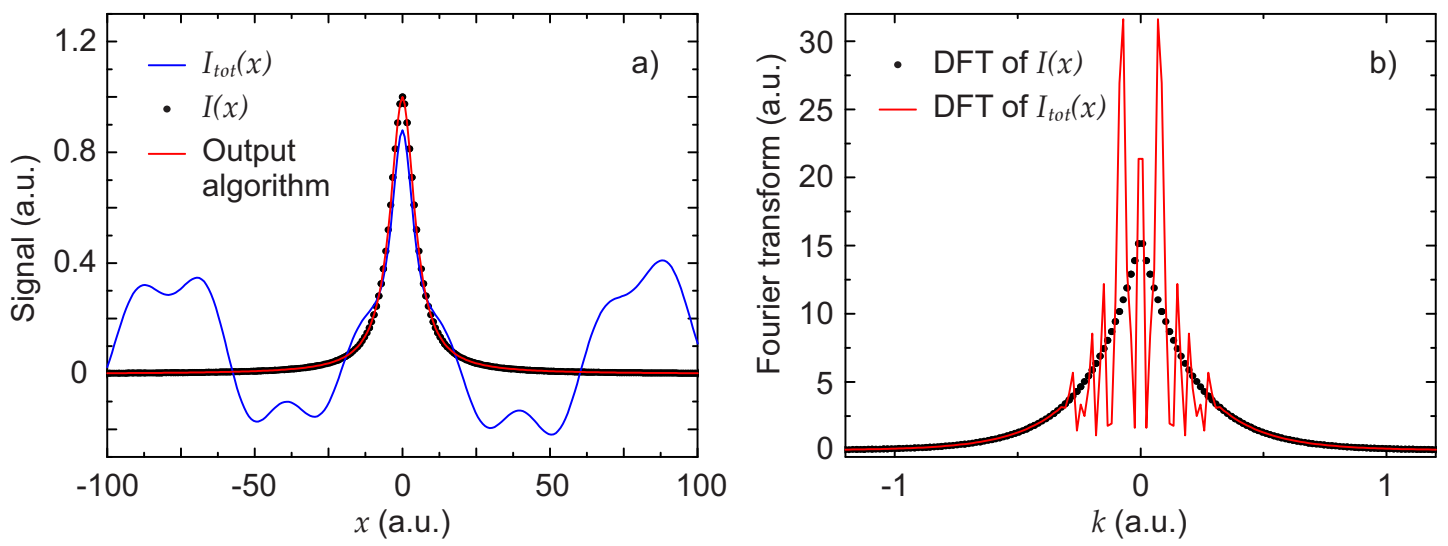

Figure 6. Third scenario: strong multiple disturbances. (a) simulated experimental total signal $I_{\text {tot }}(x)$ (blue line), Lorentzian line shape signal $I(x)$ (black dots) and extracted signal obtained with the algorithm (red line); (b) DFT of the total signal $\left|F\left(I_{t o t}(x)\right)(k)\right|$ (red line) and DFT of the Lorentzian signal $|F(I(x))(k)|$ (black points).

The percent deviation of the two parameters describing the Lorentzian obtained with the algorithm for this scenario is only of $0.012 \%$ for $P_{1}$ and $0.14 \%$ for $P_{2}$. The deviation of both parameters is particularly low in this case. To better estimate the error and the standard deviation on the parameters, the method was applied to 500 functions created with the random sum of 100 cosines described above. Then, the error was evaluated and its distribution studied. As a result, $P_{1}$ has a mean value of the absolute value of the percentage error of $0.12 \%$ with a standard deviation of $0.19 \%$, and $P_{2}$ a mean of $0.04 \%$ with a standard deviation of $0.06 \%$.

Finally, the analysis of the algorithm with simulated data has allowed for determining the causes of the discrepancy between the parameters extracted with the algorithm and those of the starting function $I(x)$. The main contribution to these arises from the approximation of the CFT by a DFT and is due to a rather large point spacing and limited $x$-range of $I(x)$ used in the simulated data. 
Smaller contributions arise from an imperfect window compensation, and a very small frequency folding [30], which was neglected here. Since the purpose of this paper is to illustrate the algorithm and not to minimize the discrepancies, the simulated data were chosen to be as close as possible to typical experimental data. The application to the three scenarios demonstrate well how, even with a very complicated background like in the third one, the proposed algorithm can extract the underlying signal extremely well.

\section{Experimental Results}

To demonstrate the robustness of the method on real gas sensing measurements, absorption spectroscopy was performed on the three strong lines R9R9 $(760.77 \mathrm{~nm})$, R7Q8 $(760.89 \mathrm{~nm})$ and R7R7 $(761.00 \mathrm{~nm})$ of the $\mathrm{O}_{2}$ near infrared A-band in the presence of multiple interference fringes.

\subsection{Experimental Setup}

The setup for the absorption spectroscopy experiments was chosen to be extremely simple and is shown schematically in Figure 7.

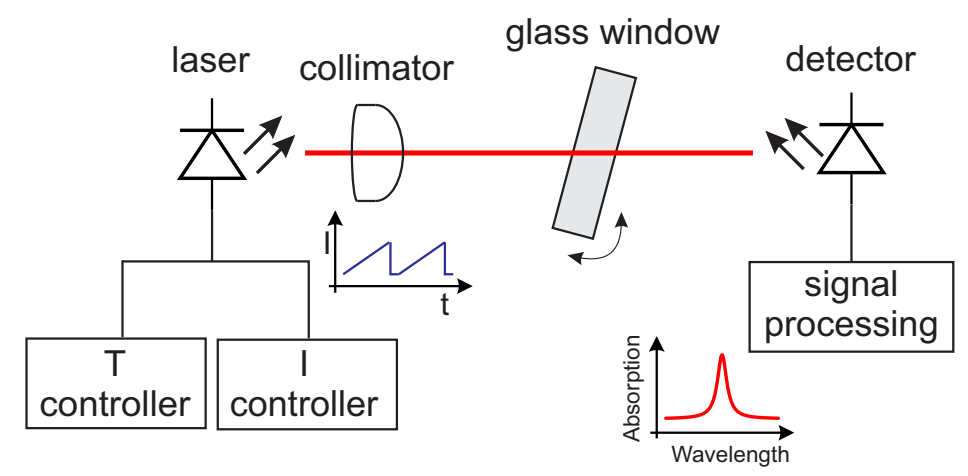

Figure 7. Schematic diagram of the setup used for the absorption spectroscopy experiments.

The light source is a $0.25 \mathrm{~mW}$ single-mode vertical-cavity surface-emitting laser (VCSEL) $(760 \mathrm{~nm}$ TO5 VCSEL, Philips Photonics, Ulm, Germany) emitting at $760 \mathrm{~nm}$. The laser current and temperature were adjusted by a temperature controller (TEC 2000, Thorlabs, Newton, NJ, USA) and a VCSEL laser diode controller (LDC 200C, Thorlabs, Newton, NJ, USA). The light emitted by the laser is collimated by a lens. The light transmitted by the sample is collected using a large-area Si $10 \times$ $10 \mathrm{~mm}^{2}$ photodiode (FS1010, Thorlabs, Newton, NJ, USA), amplified by an adjustable-gain photodiode amplifier (PDA200C, Thorlabs, Newton, NJ, USA). The current ramp for the wavelength-sweep and the data acquisition were performed by a DAQ card (USB-6361, National Instruments Switzerland GmbH, Ennetbaden, Switzerland) using a Labview ${ }^{\mathrm{TM}}$ software. The laser current sweep was chosen so to be able to measure three oxygen absorption lines. The total distance between the laser and the detector was kept fixed at approximately $36 \mathrm{~cm}$. Interference fringes of adjustable intensity were generated by inserting and tilting a glass window of known material in the optical path. By varying the thickness of the glass window, it is possible to achieve fringes with different FSR; by tilting the glass window, it is possible to adjust the amplitude of the fringes. In this work, two glass windows of BK7 of thicknesses $\mathrm{d}=11 \mathrm{~mm}$ (window 1) and $\mathrm{d}=4 \mathrm{~mm}$ (window 2) were used. These two windows were chosen to create interferences fringes with FSR comparable to (window 1) and greater than (window 2) the line width of the signal. These types of interferences are the most disturbing because they cannot be easily eliminated-for example, by a small jitter in the laser current or by standard post-processing filtering.

\subsection{Oxygen Sensing}

The absorbance signal of the three oxygen lines R9R9, R7Q8 and R7R7 is shown in Figure 8. The interference fringes due to the glass window are clearly visible. Superimposed to these 
fringes, other minor ones are also visible, which are due to the glass window of the laser package and to the surfaces of the collimator. The zero-absorbance baseline arising from the nonlinear wavelength-dependent intensity of the laser was determined by a fourth-order polynomial fit over the entire sweep range. All the measurements reported in this work were performed in air, at room temperature and ambient pressure.

Both measurements shown in Figure 8 were processed with the proposed algorithm assuming a Lorentzian line shape. The results for the line R7Q8 is shown in Figure 9. For comparison also the expected absorption lines based on the HITRAN 2012 database [31] and determined using the application SpectraPlot [32] are shown. It is evident from Figure 9, and particularly from the enlargement in (b), that the algorithm extracts the absorption line extremely well. Differently from many fitting methods, the algorithm does not require neither input parameters nor initial values. The slightly lower peak height for the line in the presence of window 1 is due to the fact that the distance between laser and detector was kept fixed and window 1 is thicker, thus reducing the optical path length for oxygen absorption of $(11-4) \mathrm{mm}=7 \mathrm{~mm}$. This difference in the HITRAN simulated curves was extracted correctly by the algorithm. To estimate the accuracy of the algorithm, the area under the absorption line from the HITRAN database calculated numerically was compared with the value of $P_{1}$ extracted with the algorithm. For both measurements, the difference is $0.1 \%$. There are still minimal deviations between the extracted and simulated lines, which could be reduced by considering a Voigt instead of a purely Lorentzian profile for the oxygen lines.

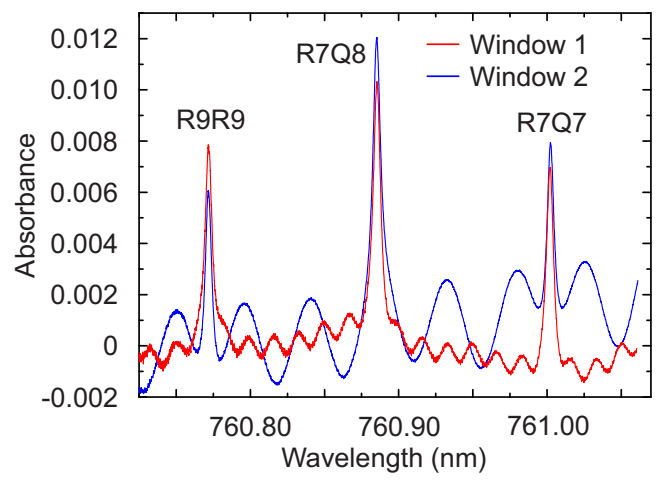

Figure 8. Typical absorbance for the three lines R9R9 (760.77 nm), R7Q8 (760.89 nm) and R7R7 $(761.003 \mathrm{~nm})$ of the $\mathrm{O}_{2}$ near infrared A-band. Superimposed to the absorption features are the interferences caused by the window $1(\mathrm{~d}=11 \mathrm{~mm})$ and window $2(\mathrm{~d}=4 \mathrm{~mm})$. The measurement is taken at atmospheric conditions.
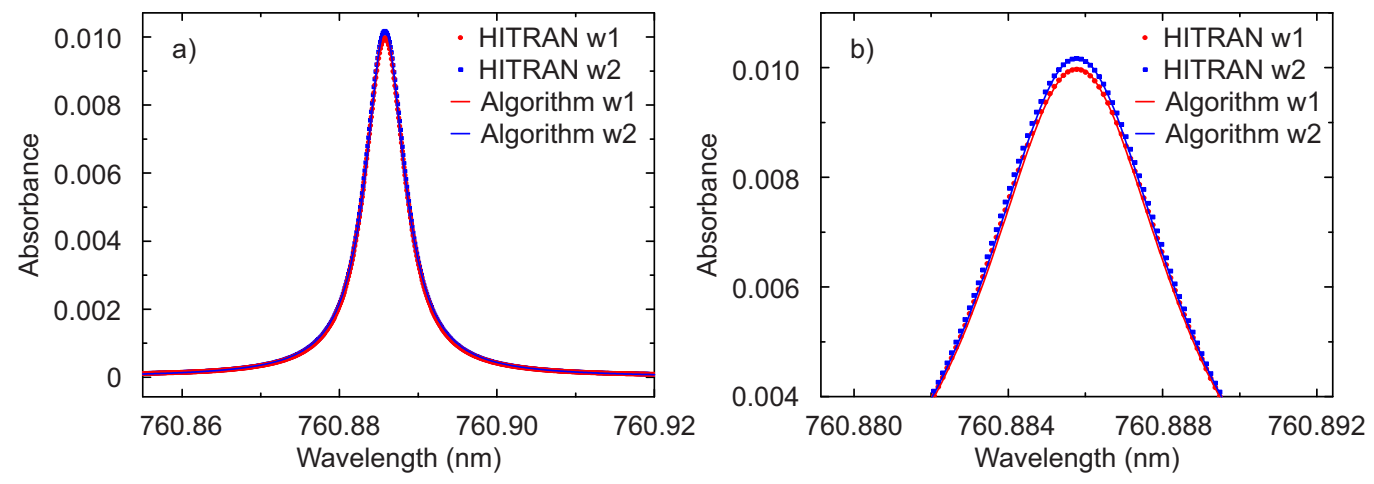

Figure 9. (a) comparison of the absorbance of the R7Q8 line extracted with the algorithm (solid lines; Algorithm w1, Algorithm w2) and the expected lines from HITRAN database (points; HITRAN w1, HITRAN w2) for the window $1(\mathrm{w} 1, \mathrm{~d}=11 \mathrm{~mm})$ and window $2(\mathrm{w} 2, \mathrm{~d}=4 \mathrm{~mm})$; (b) enlargement of the peak maximum for the same data. 


\section{Conclusions}

In this work, a novel semi-parametric algorithm is presented, which allows the extraction of a signal from an arbitrary background. In particular, the algorithm is applied to a background that is the sum of periodic interference fringes of different amplitudes and FSR. These types of disturbances, arising, for example, from multiple reflections between surfaces in the optical path, are of highest relevance for absorption spectroscopy because they are frequently the most common limit to gas sensor performance. The novel algorithm is firstly demonstrated on simulated data for three scenarios chosen to represent particularly relevant practical situations. In all three cases, the discrepancy between the results obtained with the algorithm and the expected values for the line parameters is very small-less than $0.3 \%$. Then, the algorithm is applied to experimental data of oxygen absorption in the presence of multiple interferences. Despite the strong fringes, the extracted line shows a remarkable agreement with the expected curves from the HITRAN database, with deviation of the area of only $0.1 \%$.

The great advantage of the algorithm is that it is semi-parametrical. This means that it requires no input parameters to extract the signal. This is clearly demonstrated by the third scenario with simulated data, where a background comprising a hundred cosine functions with different amplitudes, frequencies and phases could not be possibly modeled by standard nonlinear fits. In this work, the cut-off was determined dynamically by maximizing the coefficient of determination. Its recalculation at every measurement enables the accuracy achieved. This would be advantageous also in field applications, so that if the fringes should change in the time, the algorithm would automatically adjust the cut-off $i_{0}$.

These results show that the performance of a very simple sensing setup, with standard anti-reflection coatings and minimal precautions to minimize the interference fringes, can be strongly improved by simply post processing the data with the proposed algorithm.

In this paper, the algorithm was applied to a Lorentzian line shape in direct absorption spectroscopy for oxygen concentration determination. However, it can be applied to account for other line shapes, for example a Voigt profile, or to signals with a different functional form, as arising, for example, from wavelength-modulation spectroscopy. Particularly in the case of fringes with large amplitudes and FSR of the order of the FWHM of the line, it can extract the desired signal very well, making the signal insensitive to fringes and their changes with time. Preliminary results on simulated data indicate that the algorithm performs equally well even if the signal amplitude is ten times smaller than the fringes. Further studies are needed to test the performance of the algorithm in similar extreme cases.

In conclusion, the presented algorithm, being able to extract the signal feature from an arbitrary background, has the potential to allow interference-immune TDLAS, solving long-time stability problems arising from changes over time of the background, like thermal drift. Furthermore, this algorithm is not specific of TDLAS and can be applied to any kind of spectroscopic data, provided the functional shape of the signal to be detected is known.

Acknowledgments: This work was carried out under a Commission for Technology and Innovation CTI grant (17176.1 PFNM-NM).

Author Contributions: F.V. conceived, designed and performed the experiments; U.M. proposed and developed the algorithm; F.V. and U.M. analyzed the data and wrote the paper.

Conflicts of Interest: The authors declare no conflict of interest. 


\section{Abbreviations}

The following abbreviations are used in this manuscript:

TDLAS Tunable Diode Laser Absorption Spectroscopy

FSR Free Spectral Range

CFT Continuous Fourier Transform

DFT Discrete Fourier Transform

RW Rectangular Window

HWHM Half Width at Half Maximum

VCSEL Vertical-cavity surface-emitting laser

\section{Appendix A Approximation of the CFT by the Modified DFT}

In this paper, the following definition for the continuous Fourier transform (CFT) is used

$$
F(k) \equiv \int_{-\infty}^{\infty} f(x) e^{-i x k} d x
$$

Let us first make some assumptions that will simplify the calculations. Let us assume that the function $f(x)$ is zero outside a certain range $(-a / 2, a / 2)$ with some $a \in \mathbb{R}$ and $a>0$ and let us indicate with $m$ the number of data points at our disposal. For convenience, $m$ is taken to be even. Let us introduce the sampling rate $\beta$ as

$$
\beta=\frac{a}{m}
$$

The abscissas of the data $x_{j}$ and $k_{j}$ can be written as:

$$
\begin{aligned}
& x_{j}=\beta j, \\
& k_{j}=\frac{2 \pi}{a} j,
\end{aligned}
$$

with $j$ going from $-m / 2$ to $m / 2$. We can approximate Equation (A1) with a Riemann sum using the fact that $f(x)$ is zero outside the range $(-a / 2, a / 2)$ :

$$
\begin{aligned}
F\left(k_{n}\right) & =\int_{-\infty}^{\infty} f(x) e^{-i x k_{n}} d x \\
& =\int_{-a / 2}^{a / 2} f(x) e^{-i x k} d x \Longrightarrow, \\
F\left(k_{n}\right)=D_{n}(f) & \equiv \beta \sum_{j=-m / 2}^{m / 2} f\left(x_{j}\right) e^{-i x_{j} k_{n}},
\end{aligned}
$$

where we have approximated the integral with a discrete sum. Clearly, the bigger $m$ is, the better will be the approximation. Now, using Equations (A3) and (A4), we can rewrite (A5) as

$$
D_{n}(f)=\beta \sum_{j=-m / 2}^{m / 2} f\left(x_{j}\right) e^{-2 \pi i j n / m}
$$

\section{References}

1. Cassidy, D.T.; Reid, J. Atmospheric pressure monitoring of trace gases using tunable diode lasers. Appl. Opt. 1982, 21, 1185-1190.

2. Schiff, H.I.; Mackay, G.I.; Bechara, J. The use of tunable diode laser absorption spectroscopy for atmospheric measurements. Res. Chem. Intermed. 1994, 20, 525-556.

3. Fehér, M.; Martin, P.A. Tunable diode laser monitoring of atmospheric trace gas constituents. Spectrochim. Acta A 1995, 51, 1579-1599. 
4. Fried, A.; Henry, B.; Wert, B.; Sewell, S.; Drummond, J.R. Laboratory, ground- based and airborne tunable diode laser systems performance characteristics and applications in atmospheric studies. Appl. Phys. B 1998, $67,317-330$.

5. Tuzson, B.; Henne, S.; Brunner, D.; Steinbacher, M.; Mohn, J.; Buchmann, B.; Emmenegger, L. Continuous isotopic composition measurements of tropospheric CO2 at Jungfraujoch (3580 m a.s.l.), Switzerland: Real-time observation of regional pollution events. Atmos. Chem. Phys. 2011, 11, 1685-1696.

6. Nikodem, M.; Wysocki, G. Chirped Laser Dispersion Spectroscopy for Remote Open-Path Trace-Gas Sensing. Sensors 2012, 12, 16466-16481.

7. McCurdy, M.R.; Bakhirkin, Y.; Wysocki, G.; Lewicki, R.; Tittel, F.K. Recent advances of laser-spectroscopy based techniques for applications in breath analysis. J. Breath Res. 2007, 1, 014001.

8. Wang, C.; Sahay, P. Breath Analysis using laser spectroscopic techniques: Breath biomarkers, spectral fingerprints, and detection limits. Sensors 2009, 9, 8230-8262.

9. Risby, T.H.; Tittel, F.K. Current status of mid-infrared quantum and interband cascade lasers for clinical breath analysis. Opt. Eng. 2010, 49, 111123/1-24.

10. Curl, R.F.; Capasso, F.; Gmachl, C.; Kosterev, A.A.; McManus, B.; Lewicki, R.; Pusharsky, M.; Wysocki, G.; Tittel, F.K. Quantum cascade lasers in chemical physics. Chem. Phys. Lett. 2010, 487, 1-20.

11. Linnerud, I.; Kaspersen, P.; Jaeger, T. Gas monitoring in the process industry using diode laser spectroscopy. Appl. Phys. B 1998, 67, 297-305.

12. Lackner, M. Tunable diode laser absorption spectroscopy (TDLAS) in the process industries-A review. Rev. Chem. Eng. 2007, 23, 65-147.

13. Kluczynski, P.; Jahjah, M.; Nähle, L.; Axner, O.; Belahsene, S.; Fischer, M.; Koeth, J.; Rouillard, Y.; Westberg, J.; Vicet, A.; et al. Detection of acetylene impurities in ethylene and polyethylene manufacturing processes using tunable diode laser spectroscopy in the 3- $\mu \mathrm{m}$ range. Appl. Phys. B 2011, 105, 427-434.

14. Hodgkinson, J.; Tatam, R.P. Optical gas sensing: A review. Meas. Sci. Technol. 2013, 24, doi:10.1088/0957-0233/24/1/012004.

15. Masiyano, D.; Hodgkinson, J.; Schilt, S.; Tatam, R.P. Self-mixing interference effects in tunable diode laser absorption spectroscopy. Appl. Phys. B 2009, 96, 863-874.

16. Hartmann, A.; Strzoda, R.; Schrobenhauser, R.; Weigel, R. Ultra-compact TDLAS humidity measurement cell with advanced signal processing. Appl. Phys. B 2014, 115, 263-268.

17. Werle, P. Accuracy and precision of laser spectrometers for trace gas sensing in the presence of optical fringes and atmospheric turbulence. Appl. Phys. B 2011, 102, 313-329.

18. Webster, C. Brewster-plate spoiler: A novel method for reducing the amplitude of interference fringes that limit tunable-laser absorption sensitivities. J. Opt. Soc. Am. B 1985, 2, 1464-1470.

19. Silver, J.A.; Stanton, A.C. Optical interference fringe reduction in laser absorption experiments. Appl. Opt. 1988, 27, 1914-1916.

20. Whittaker, E.A.; Gehrtz, M.; Bjorklund, G. Residual amplitude modulation in laser electro-optic phase modulation. J. Opt. Soc. Am. B 1985, 2, 1320-1326.

21. Sun, H.C.; Whittaker, E.A. Novel etalon fringe rejection technique for laser absorption spectroscopy. Appl. Opt. 1992, 31, 4998-5002.

22. Goldenstein, C.S.; Strand, C.L.; Schultz, I.A.; Sun, K.; Jeffries, J.B.; Hanson, R.K. Fitting of calibration-free scanned-wavelength-modulation spectroscopy spectra for determination of gas properties and absorption lineshapes. Appl. Opt. 2014, 53, 356-367.

23. Ehlers, P.; Johansson, A.C.; Silander, I.; Foltynowicz, A.; Axner, O. Use of etalon-immune distances to reduce the influence of background signals in frequency-modulation spectroscopy and noise-immune cavity enhanced optical heterodyne molecular spectroscopy. J. Opt. Soc. Am. B 2014, 31, 2934-2945.

24. Chen, J.; Hangauer, A.; Strzoda, R.; Amann, M.C. Laser spectroscopic oxygen sensor using diffuse reflector based optical cell and advanced signal processing. Appl. Phys. B 2010, 100, 417-425.

25. Li, J.; Yu, B.; Zhao, W.; Chen, W. A review of signal enhancement and noise reduction techniques for tunable diode laser absorption spectroscopy. Appl. Spectrosc. Rev. 2014, 49, 666-691.

26. Tenoudji, F.C. Analog and Digital Signal Analysis, 1st ed.; Springer International Publishing: Cham, Switzerland, 2016; pp. 111-113.

27. Tukey, J.W. An introduction to the calculations of numerical spectrum analysis. In Spectral Analysis of Time Series; Wiley: New York, NY, USA, 1967; pp. 25-46. 
28. Harris, F.J. On the use of Windows for Harmonic Analysis with the Discrete Fourier Transform. Proc. IEEE 1978, 66, 51-83.

29. Reid, J.; El-Sherbiny, M.; Garside, B.K.; Ballik, E.A. Sensitivity limits of a tunable diode laser spectrometer, with application to the detection of $\mathrm{NO}(2)$ at the 100-ppt level. J. Appl. Opt. 1980, 19, 3349-3354.

30. Tenoudji, F.C. Analog and Digital Signal Analysis, 1st ed.; Springer International Publishing: Cham, Switzerland, 2016; pp. 265-267.

31. Rothman, L.S.; Gordon, I.E.; Babikov, Y.; Barbe, A.; Chris Benner, D.; Bernath, P.F.; Birk, M.; Bizzocchi, L.; Boudon, V.; Brown, L.R.; et al. The HITRAN2012 molecular spectroscopic database. J. Quant. Spectrosc. Radiat. Transf. 2013, 130, 4-50.

32. SpectraPlot the Wavelength Search Engine. Available online: http:/ /www.spectraplot.com (accessed on 1 April 2017).

(C) 2017 by the authors. Licensee MDPI, Basel, Switzerland. This article is an open access article distributed under the terms and conditions of the Creative Commons Attribution (CC BY) license (http://creativecommons.org/licenses/by/4.0/). 